\title{
ON THE LOCAL TIME DENSITY OF THE REFLECTING BROWNIAN BRIDGE
}

\author{
BERNHARD GITTENBERGER ${ }^{1}$ \\ TU Wien, Department of Geometry \\ Wiedner Hauptstrasse 8-10/113, A-1040, Wien, Austria \\ E-mail: gittenberger@tuwien.ac.at \\ GUY LOUCHARD \\ Université Libre de Bruxelles \\ Départemente d'Informatique, -C.P. 212, Campus de la Plaine \\ Boulevard du Triompher, B-1050 Bruxelles, Belgium \\ E-mail: louchard@ulb.ac.be
}

(Received March, 1999; Revised September, 1999)

Expressions for the multi-dimensional densities of Brownian bridge local time are derived by two different methods: A direct method based on Kac's formula for Brownian functionals and an indirect one based on a limit theorem for strata of random mappings.

Key words: Brownian Bridge, Local Time, Random Trees.

AMS subject classifications: $60 \mathrm{~J} 55,60 \mathrm{C} 05$.

\section{Introduction}

Throughout this paper, the standard Brownian motion (BM) will be denoted by $x(t)$. The reflecting Brownian bridge (rBB) is the process $x^{+}(t)$, which is identical in law to $(|x(t)-t x(1)|, 0 \leq t \leq 1)$.

We are interested in the process $\left(\tau^{+}(a), a \geq 0\right)$, where $\tau^{+}(a)$ is the total local time of $x^{+}(t)$ at level $a$, defined by

$$
\tau_{+}(a)=\lim _{\varepsilon \rightarrow 0} \frac{1}{\varepsilon} \int_{0}^{1} I_{[a, a+\varepsilon]}\left(x^{+}(t)\right) d t .
$$

Several representations of the one-dimensional density of this process are known. Though there is no direct study of this process, results on symmetric random walks or random mappings $([2,7])$ yield various density representations (see $[21,22,7])$.

\footnotetext{
${ }^{1}$ This author's work was supported by the Austrian Science Foundation FWF, grant P10187-PHY, and the Stiftung Aktion Österreich-Ungarn grant 34oeu24.
} 
Apart from the random mapping (see Section 2.3), applications of the rBB local time can be found in the analysis of Shellsort (see Louchard [17, §2]). We need the distribution of the number $I(2 n)$ of inversions in a 2-ordered permutation of the $2 n$ values $\{1 \ldots 2 n\}$ (i.e., permutation consisting of two interleaved sorted permutations). Position $i$ of the odd part of the permutation contains value $k$ if the path $U_{n}$ corresponding to the permutation satisfies $U_{n}(i)=k-i$ (this is Knuth's correspondence between 2-ordering and path in a lattice, see Knuth $\left[15\right.$, p. 87]). Now $U_{n}([n t]-$ $n t) / \sqrt{2 n} \Rightarrow B B(t)$ and $I(2 n)=\sum_{i=1}^{n}\left|i-U_{n}(i)-1\right|$.

The local time of the rBB at $\ell / \sqrt{2 n}$ corresponds to the number of positions $i$ containing $2 i+\ell$ or $2 i-\ell$.

The rBB local time corresponds also to the number of jumps at some level of the empirical distribution, in the context of the classical Kolmogorov-Smirnov distribution function.

Denote by $f_{x}(y)$ the density of $\tau^{+}(x)$. Then in [7] we find the representation

$$
f_{x}(y)=\frac{1}{i \sqrt{2 \pi}} \int_{S} \frac{e^{\alpha}}{2 \cosh ^{2}(x \sqrt{2 \alpha})} \exp \left(-\frac{y}{\sqrt{2}} \frac{\sqrt{\alpha} e^{x \sqrt{2 \alpha}}}{\cosh (x \sqrt{2 \alpha})}\right) d \alpha
$$

where $S:=(1-i \infty, a+i \infty), a>0$, is a straight line parallel to the imaginary axis, which is the Brownian bridge analog to the density presented in [5, 13] for the Brownian excursion. We will generalize this formula to several dimensions and offer two approaches: The first one is a direct computation by means of Kac's formula for Brownian functionals and the second one is based on the fact that the process consisting of the - suitably normalized - strata of a random mapping converges weakly to Brownian bridge local time.

The paper is organized as follows. In Section 2, we summarize basic notations and methods. Some preliminary formulas based on Kac's formula and their inversion are given in Section 3. Section 4 is devoted to the general multi-dimensional density. The Brownian excursion analog of this problem has been treated in [12]. Thus we keep our presentation rather brief and refer to [12] for details.

We would like to mention that MAPLE was of great help in computing some complicated expressions (with some guidance, of course).

\section{Basic Notations and Known Results}

\subsection{Kac's formula for Brownian functionals}

Denote by $\eta(t)$ any of the processes defined in the previous section. Then we will use the notation

$$
E_{a}[B(\eta)]:=\operatorname{Pr}[B \mid \eta(0)=a]
$$

where $B(\eta)$ is an event belonging to the Borel field generated by $\eta(t)$. Furthermore, denote by $\mathcal{L}_{\alpha}(f(x)):=\int_{0}^{\infty} e^{-\alpha x} f(x) d x$, the Laplace transform of $f(x)$. Then the classical density (for $\eta(t)=x(t)$ )

$$
p(t, x, y) d y:=E_{x}[x(t) \in d y]=\frac{1}{\sqrt{2 \pi t}} \exp \left[-\frac{(x-y)^{2}}{d t}\right] d y
$$


implies

$$
\ell_{\alpha}(p(t, x, y))=\frac{\exp (-\sqrt{2 \alpha}|x-y|)}{\sqrt{2 \alpha}}
$$

where the Laplace transform is taken with respect to $t$.

Let $h \geq 0$ be a piecewise continuous function and let $g$ be the differential operator

$$
(\mathrm{g} u)(a):=\frac{1}{2} u^{\prime \prime}(a)-h(a) u(a)
$$

Kac's formula states that, for $\alpha>0$ and $f \in C\left(R^{1}\right)$,

$$
u(a)=E_{a} \int_{0}^{\infty} e^{-\alpha t} \exp \left(-\int_{0}^{t} h[x(s)] d s\right) f(x(t)) d t
$$

is the bounded solution of

$$
(\alpha-\mathrm{g}) u=f
$$

The solution of (4) is given by $u(a)=\int G(a, b) f(b) d b$ where the Green function $G$ is given by

$$
G(a, b)=G(b, a)=2 W^{-1} g_{1}(a) g_{2}(b), a \leq b,
$$

where $0<g_{1} \in \uparrow, 0<g_{2} \in \downarrow$ are independent solutions of $g g=\alpha g$ and $W$ is their constant positive Wronskian: $W=g_{1}^{\prime} g_{2}-g_{1} g_{2}^{\prime}$ (see Itô and McKean [14, par. 2.6] and Louchard [18]).

In (3), if we add another function $h^{*}$ to $h$, the modified Green function $G^{*}(a, b)$ satisfies the relation

$$
G^{*}(a, b)=G(a, b)-\int G(a, x) h^{*}(x) G^{*}(x, b) d x
$$

(see [14, p. 67]).

Particularly, for $h^{*}(x)=\gamma I_{(\xi, \eta)}(x) /(\eta-\xi)$ (where $I_{(\xi, \eta)}$ is the indicator function of the interval $(\xi, \eta)$ with $\eta>\xi)$ we obtain, letting $\eta \downarrow \xi$

$$
G^{*}(a, b)=G(a, b)-\gamma \frac{G(a, \xi) G(\xi, b)}{1+\gamma G(\xi, \xi)} .
$$

This corresponds to adding $\gamma t^{+}(t, \xi)$ to $\int_{0}^{t} h[x(s)] d s$. Letting $\gamma \uparrow \infty$, we get from (5)

$$
G(a, b)-\frac{G(a, \xi) G(\xi, b)}{G(\xi, \xi)}
$$

which corresponds to

$$
\int_{0}^{\infty} e^{-\alpha t} E_{a}\left[\exp \left[-\int_{0}^{t} h[x(s)] d s\right], t<m_{\xi}, x(t) \in d b\right] d t \quad a, b<\xi
$$

where $m_{a}(\eta):=\inf (s: \eta(s)=a)$ denotes the hitting time. 


\subsection{Random mappings and local time}

As usual, a random mapping on the set $\{1, \ldots, n\}$ is defined to be an element of the set $F_{n}$ of all mappings $\varphi:\{1, \ldots, n\} \rightarrow\{1, \ldots, n\}$ equipped with the uniform distribution. It can be represented by its functional graph $G_{\varphi}$, i.e., the graph consisting of the nodes $1,2, \ldots, n$ and of the edges $(i, \varphi(i)), i=1, \ldots, n$. It is easy to see that each component of such a graph consists of exactly one cycle of length $\geq 1$, each point of which is the root of a labeled tree (a so-called Cayley tree). Thus for each point $x \in$ $G_{\varphi}$, there exists a unique path connecting $x$ with the next cyclic point. The length of this path is called the distance of $x$ to the cycle. The set of all points at a fixed distance $r$ from the cycle is often called the $r$ th stratum of $\varphi$.

Let $L_{n}(t)$ denote the number of nodes in the $t$ th stratum of a random mapping $\varphi \in F_{n}$. For noninteger $t$, define

$$
L_{n}(t)=(\lfloor t\rfloor+1-t) L_{n}(\lfloor t\rfloor)+(t-\lfloor t\rfloor+1), \quad t \geq 0 .
$$

There is a lot of literature on random mappings and interested readers should consult [16]. In the sequel, we will need the following results from [7].

Theorem 2.1: The following limit theorem holds in $C[0, \infty)$ :

$$
\frac{1}{\sqrt{n}} L_{n}(t n \sqrt{n}) \stackrel{w}{\rightarrow} \frac{1}{2} \tau+\left(\frac{t}{2}\right)
$$

in $C[0, \infty)$ as $n \rightarrow \infty$.

By means of this theorem, we will compute the multi-dimensional local time densities: Let $a_{n}=n^{n-1}$ be the number of Cayley trees consisting of $n$ nodes. Furthermore, denote by $a_{k_{1} \ldots d_{d}}^{\left(r_{1} \ldots r_{d}\right)}$, the number of all random mappings in $F_{n}$ which have $k_{i}$ nodes in stratum $r_{i}$, where $r_{1}<\ldots<r_{d}$. Then by setting

$$
\begin{gathered}
b_{0}(z, u)=u \\
b_{i+1}(z, u)=z e^{b_{i}(z, u)}
\end{gathered}
$$

and

$$
a(z)=\sum_{n \geq 0} a_{n} \frac{z^{n}}{n !}
$$

and using standard combinatorial techniques (readers not familiar with these techniques may consult [10] and [8]), we can write down the generating function of these numbers in the form

$$
\begin{gathered}
g\left(z, u_{1}, \ldots, u_{d}\right)=\sum_{k_{1}, \ldots, k_{d}, n \geq 0} a_{k_{1} \cdots k_{d}^{n}}^{\left(r_{1} \ldots r_{d}\right) u_{1}^{k} 1 \ldots u_{d}^{k} z^{n}} \\
=\frac{1}{1-b_{r_{1}}\left(z, u_{1} b_{h_{12}}\left(z, u_{2} b_{h_{23}}\left(\cdots u_{d-1} b_{h_{d-1, d}}\left(z, u_{d} a(z)\right) \cdots\right)\right)\right.}
\end{gathered}
$$

where $h_{i j}=r_{j}-r_{i}$.

The multi-dimensional density can be determined by evaluating the proper coefficient of $g\left(z, u_{1}, \ldots, u_{d}\right)$. If we set $r_{i}=\left\lfloor\rho_{1} \sqrt{n}\right\rfloor$ and $k_{i}=\left\lfloor y_{i} \sqrt{n}\right\rfloor$ and denote by $\underset{\text { have }}{f_{\rho_{1} \ldots \rho_{d}}}\left(y_{1}, \ldots, y_{d}\right)$ the joint density of $\left(\tau^{+}\left(\rho_{1}\right), \ldots, \tau^{+}\left(\rho_{d}\right)\right)$, then by Theorem 2.1 we 


$$
f_{\rho_{1} / 2, \ldots, \rho_{d} / 2}\left(2 y_{1}, \ldots, 2 y_{d}\right)=2^{-d} \lim _{n \rightarrow \infty} \frac{n^{d / 2} n !}{n^{n}}\left[z^{n} u_{1}^{k} 1 \ldots u_{d}^{k} d g\left(z, u_{1}, \ldots, u_{d}\right)\right.
$$

where the symbol $\left[z^{n}\right] f(z)$ denotes the coefficient of $z^{n}$ in the power series expansion of $f(z)$.

\section{Preliminary Formulas}

In this section, we define some auxiliary functions built on Kac's formula. Let $\left(\rho_{i}\right)$ be a strictly monotonically increasing sequence of non-negative real numbers. Set

$$
\varphi_{d}(\alpha):=\int_{0}^{\infty} e^{-\alpha t} E\left[e^{\left.-\beta_{1} \sqrt{t} \tau+\left(\rho_{1} / \sqrt{t}\right)-\ldots-\beta_{d} \sqrt{t} \tau+\left(\rho_{d} / \sqrt{t}\right)\right]} \frac{d t}{\sqrt{2 \pi t}}\right.
$$

and

$$
\begin{aligned}
& \bar{\varphi}_{d}(\alpha):= \\
& \int_{0}^{\infty}\left[e^{-\alpha t}-1\right] E\left[e^{-\beta_{1} \sqrt{t}+\left(\rho_{1} / \sqrt{t}\right)-\ldots-\beta_{d-1} \sqrt{t}+\left(\rho_{d-1} / \sqrt{t}\right)}\left[t<m_{\rho_{d}}\right]\right] \frac{d t}{\sqrt{2 \pi t}}
\end{aligned}
$$

where $m$ is related to the Brownian bridge of duration $t$.

Lemma 3.1: We have

$$
\varphi_{d}(\alpha)=\left.G_{d}^{* *}(a, b)\right|_{a=b=0}
$$

and

$$
\bar{\varphi}_{d}(\alpha)=\left.\bar{G}_{d}^{* *}(a, b)\right|_{a=b=0},
$$

where

$$
\begin{aligned}
& G_{d}^{* *}(a, b)=G_{d}^{*}(a, b)-\beta_{d} \frac{G_{d}^{*}\left(a,-\rho_{d}\right)\left(G_{d}^{*}\left(-\rho_{d}, b\right)\right.}{1+\beta_{d} G_{d}^{*}\left(-\rho_{d},-\rho_{d}\right)} \\
& \bar{G}_{d}^{* *}(a, b)=\bar{G}_{d}^{*}(a, b)-\beta_{d} \frac{\bar{G}_{d}^{*}\left(a,-\rho_{d}\right)\left(\bar{G}_{d}^{*}\left(-\rho_{d}, b\right)\right.}{\bar{G}_{d}^{*}\left(-\rho_{d},-\rho_{d}\right)}
\end{aligned}
$$

with

$$
G_{d}^{*}(a, b)=\left\{\begin{array}{cl}
G(a, b)-\beta_{1} \frac{G\left(a, \rho_{1}\right) G\left(\rho_{1}, b\right)}{1+\beta_{1} G\left(\rho_{1}, \rho_{1}\right)} & \text { for } d=1 \\
G_{d-1}^{* *}(a, b)-\beta_{d} \frac{G_{d-1}^{* *}\left(a, \rho_{d}\right) G_{d-1}^{* *}\left(\rho_{d}, b\right)}{1+\beta_{d} G_{d-1}^{* *}\left(\rho_{d}, \rho_{d}\right)} & \text { for } d>1
\end{array}\right.
$$

and

$$
\bar{G}_{d}^{*}(a, b)=\left\{\begin{array}{cl}
G(a, b)-\frac{G\left(a, \rho_{1}\right) G\left(\rho_{1}, b\right)}{G\left(\rho_{1}, \rho_{1}\right)} & \text { for } d=1 \\
G_{d-1}^{* *}(a, b)-\frac{G_{d-1}^{* *}\left(a, \rho_{d}\right) G_{d-1}^{* *}\left(\rho_{d}, b\right)}{G_{d-1}^{* *}\left(\rho_{d}, \rho_{d}\right)} & \text { for } d>1
\end{array}\right.
$$


and

$$
G(a, b)=\frac{\exp (-\sqrt{2 \alpha}|a-b|)}{\sqrt{2 \alpha}}
$$

Proof: By (2) we have

$$
G(a, b) d b=\int_{0}^{\infty} e^{-\alpha t} E_{a}[x(t) \in d b] d t
$$

Inserting $h \equiv 0$ and $f(y)=I_{d b}(y)$ into Kac's formula and adding $\gamma t^{+}(t, \rho)$ as described before, formula (6) yields

$$
\begin{aligned}
G_{1}(a, b) d b & =\lim _{\gamma \uparrow \infty} \int_{0}^{\infty} e^{-\gamma t} E_{a}\left[\exp \left(-\gamma t^{+}(t, \rho)\right), x(t) \in d b\right] d t \\
& =\int_{0}^{\infty} e^{-\alpha t} E_{a}\left[x(t) \in d b, t<m_{\rho}\right] d t
\end{aligned}
$$

Adding a local time $\beta t^{+}(t, \rho)$ to the exponent gives a modification to the Green function according to (5) and thus we have

$$
G_{2}(a, b)=\int_{0}^{\infty} e^{-\alpha t} E_{a}\left[e^{-\beta t+(t, \rho)} x(t) \in d b\right] d t
$$

Continuing in this way (considering $\rho_{d},-\rho_{d}$ ) yields recursion (12) and (11). For obtaining (13), we have to take into account that we restrict to the $d$ th step $\left[t<m_{\rho_{d}}\right]$ and $\left[t<m_{-\rho_{d}}\right]$ (this way we obtained (5) and (6), i.e., by adding $\gamma t^{+}\left(t, \rho_{d}\right)$ and $\gamma t^{+}\left(t,-\rho_{d}\right)$ to the exponent and letting $\gamma \uparrow \infty$ instead of adding the local time $\left.\beta_{1} t^{+}\left(t, \rho_{1}\right)\right)$.

Examples: Dimension 0. $\quad \bar{\varphi}_{1}(\alpha)=\frac{S h\left(\sqrt{2 \alpha} x_{1}\right.}{\sqrt{2 \alpha} C h\left(\sqrt{2 \alpha} x_{1}\right)}$ : it is correct and corresponds to the Kolmogorov-Smirnov formula.

\section{Dimension 1:}

$$
\begin{gathered}
\varphi_{1}(\alpha)-\bar{\varphi}_{1}(\alpha)=\frac{2 e^{2 \sqrt{2 \alpha} x_{1}}}{\left(1+e^{2 \sqrt{2 \alpha} x_{1}}\right)\left(\sqrt{2 \alpha} e^{2 \sqrt{2 \alpha} x_{1}}+\beta_{1}\left[1+e^{2 \sqrt{2 \alpha} x_{1}}\right]\right)} \\
=\frac{1}{C h\left(\sqrt{2 \alpha} x_{1}\right)\left[\sqrt{2 \alpha} e^{\sqrt{2 \alpha} x_{1}}+2 \beta_{1} C h\left(\sqrt{2 \alpha} x_{1}\right)\right]} .
\end{gathered}
$$

Inverting, this leads to (1).

Dimension 2:

with

$$
\varphi_{2}(\alpha)-\bar{\varphi}_{2}(\alpha)=\frac{\alpha}{2 f_{3}^{2}\left[\beta_{2}+\frac{\sqrt{\alpha} E^{2,1}}{\sqrt{2} S h_{2,1}}+\frac{f_{5}}{f_{3}}\right]}
$$




$$
\begin{gathered}
f_{5}:=-\alpha \frac{C h_{1}}{S h_{2,1}} \\
f_{3}=\sqrt{2} C h_{1} S h_{2,1}\left[\beta_{1}+\frac{\sqrt{\alpha} C h_{2}}{\sqrt{2} C h_{1} S h_{2,1}}\right] \\
C h_{1}:=\cosh \left(\sqrt{2 \alpha} \rho_{1}\right) \\
S h_{2,1}:=\sinh \left(\sqrt{2 \alpha}\left(\rho_{2}-\rho_{1}\right)\right) \\
E^{2,1}:=e^{\sqrt{2 \alpha}\left(\rho_{2}-\rho_{1}\right)} .
\end{gathered}
$$

Inverting leads to

$$
\begin{aligned}
& \frac{\alpha}{4 C h_{1}^{2} S h_{2,1}^{2}} e^{\frac{-y_{1} \sqrt{\alpha} C h_{2}}{\sqrt{2} C h_{1} S h_{2,1}}-y_{2} \frac{\sqrt{\alpha} E^{2,1}}{\sqrt{2} S h_{2,1}}} \sqrt{\frac{y_{1}}{a_{1}}} I_{1}\left(2 \sqrt{a_{1} y_{1}}\right) \\
& a_{1}=\frac{\alpha y_{2}}{2 S h_{2,1}^{2}} .
\end{aligned}
$$

In order to get our density representations we have to invert the formulas. This is

done by the following.
Lemma 2: Set $\mathscr{B}_{d}(t):=e^{-\beta_{1} \sqrt{t} \tau+\left(\rho_{1} / \sqrt{t}\right) \ldots-\beta_{d} \sqrt{t} \tau+\left(\rho_{d} / \sqrt{t}\right)}$. We obtain the following inversion formulas:

$$
E\left[\mathscr{B}_{d}(1), 1>m_{\rho_{d}}\right]=\frac{1}{\sqrt{2 \pi} i} \int_{S} e^{u}\left[\Psi_{d}(u)-\Psi_{d}(u)\right] d u .
$$

Proof: See [12, Section 4].

\section{Multi-dimensional Densities}

We offer two different proofs of our results: The first one is based on some properties of $G_{d}^{* *}$. The other one is based on Cauchy's formula applied to (8) and singularity analysis.

Lemma 3: We have

(A) $\varphi_{d}(\alpha)-\bar{\varphi}_{d}(\alpha)=\Theta(d)$ with

$$
\Theta(d):=\frac{\alpha^{d-1}}{2\left[F_{1}(d)\right]^{2}\left[\beta_{d}+C_{1}(d)+C_{2}(d) D_{2}(d) / F_{1}(d)\right]}
$$

where

$$
\begin{gathered}
C_{1}(d)=\sqrt{\frac{\alpha}{2}} E^{d, d-1} / S h_{d, d-1} \\
C_{2}(d)=-\frac{\alpha}{2 S h_{d, d-1}^{2}}
\end{gathered}
$$




$$
\begin{gathered}
C_{3}(d)=\sqrt{\frac{\alpha}{2 S h_{d, d-1} S h_{d-1, d-2}}} \\
F_{1}(d)=\beta_{d-1} D_{2}(d)+D_{1}(d) \\
D_{2}(d)=\left(\beta_{d-2} D_{2}(d-1)+D_{1}(d-1)\right) \sqrt{2} S h_{d, d-1} \\
=\sqrt{2} S h_{d, d-1} F_{1}(d-1) \\
D_{1}(d)=C_{3}(d) D_{2}(d)+C_{2}(d-1) D_{2}(d-1) \sqrt{2} S h_{d, d-1} \\
=\sqrt{2} S h_{d, d-1} C_{3}(d) F_{1}(d-1)+2 S h_{d, d-1} S h_{d-1, d-2} C_{2}(d-1) F_{1}(d-2) .
\end{gathered}
$$

The coefficient of $\beta_{1} \ldots \beta_{d-2}$ in $D_{2}(d)$ equals $w^{(d-1) / 2} C h_{1} \prod_{l=2}^{d} S h_{l, l-1}$ or, equivalently, the initial values of $F_{1}(d)$ are given by

$$
\begin{gathered}
F_{1}(1)=C h_{1} \quad F_{1}(2)=\sqrt{2} C h_{1} S h_{2,1}\left[\beta+\frac{\sqrt{2} C h_{2}}{\sqrt{2} C h_{1} S h_{2,1}}\right] \\
\text { where } \quad S h_{i, j}=\sinh \left(\sqrt{2 \alpha}\left(\rho_{i}-\rho_{j}\right)\right), \quad C h_{i, j}=\cosh \left(\sqrt{2 \alpha}\left(\rho_{i}-\rho_{j}\right)\right), \quad \text { and } \\
E^{i, j}=e^{\sqrt{2 \alpha}\left(\rho_{i}-\rho_{j}\right)} .
\end{gathered}
$$

(B)

$$
\bar{\varphi}_{d}(\alpha)-\bar{\varphi}_{d-1}(\alpha)=\Theta(d-1)-\frac{\alpha^{d-1}}{2\left[F_{1}(d)\right]^{2}\left[C_{1}(d)+C_{2}(d) D_{2}(d) / F_{1}(d)\right]}
$$

Once we have proved Lemma 3, it is now routine to derive the following theorem: Part $\mathrm{A}$ is computed as in [12]. In part $\mathrm{B}$, we use the transforms

$$
\ell_{\alpha}\left[\frac{1}{a}\left(1-e^{a x}\right)\right]=\frac{1}{\alpha(\alpha+a)} .
$$

Theorem 4.1: $(A)$ The d-dimensional density is given by

$$
\begin{gathered}
f_{\rho_{1} \ldots \rho_{d}}\left(y_{1}, y_{2}, \ldots, y_{d} ; M>\rho_{d}\right) \\
=\frac{1}{i \sqrt{2 \pi}} \int_{S} \frac{e^{\alpha} \alpha^{d-1}}{2^{d} C h_{1}^{2} \prod_{l=2}^{d} S h_{l, l-1}^{2}} \\
\cdot \exp \left[-y_{d} C_{1}(d)-\sum_{l=2}^{d-1} y_{l} C_{3}(l+1)-y_{1} \frac{\sqrt{\alpha} C h_{2}}{\sqrt{2} C h_{1} S h_{2,1}}\right] \\
\cdot \prod_{l=1}^{d-1}\left[\sqrt{\frac{y_{l}}{a_{l} y_{l+1}}} I_{1}\left[2 \sqrt{a_{l} y_{l} y_{l+1}}\right]\right] d \alpha
\end{gathered}
$$

with $M=\sup _{u \in[0,1]}[Y(u) \mid L=1]$ and $a_{l}:=-\frac{\alpha}{2 S h_{l}^{2}+1, l}$.

(B) The constraint densities $f_{\rho_{1} \ldots \rho}\left(y_{1}, \ldots, y_{k} ; \rho_{k+1}>M>\rho_{k}\right)$ are given by (14) where we replace $d$ by $k$ and $-y_{k} \ddot{C}_{1}(k)$ by $-y_{k} C_{3}(k+1)$. 


\subsection{The Proofs}

\subsubsection{Using some properties of $G_{d}^{* *}$}

Proof of Lemma 3.3 Part $(\boldsymbol{A})$ : Actually, we will use the same notation as in the proof of Lemma 3 in [12] and use auxiliary functions $D_{3}(d), D_{4}(d)$, for which we will prove the following relations:

$$
\begin{gathered}
D_{2}(d)=\beta_{d-2} D_{4}(d)+D_{3}(d) \\
\frac{D_{1}(d)}{D_{2}(d)}=C_{3}(d)+C_{4}(d) D_{4}(d) / D_{2}(d) \\
C_{4}(d)=C_{2}(d-1) \\
\frac{D_{3}(d)}{D_{4}(d)}=\frac{D_{1}(d-1)}{D_{2}(d-1)} \\
D_{4}(d)=D_{2}(d-1) \sqrt{2} S h_{d, d-1}
\end{gathered}
$$

The coefficient of $\beta_{1} \ldots \beta_{d-3}$ in $D_{4}(d)=2^{(d-1) / 2} C h_{1} \prod_{l=2}^{d} S h_{l, l-1}$.

By (12) and (13) we have

$$
\begin{gathered}
G_{d}^{* *}(a, b)-\bar{G}_{d}^{* *}(a, b)=\frac{2 G_{d-1}^{* *}\left(a, \rho_{d}\right) G_{d-1}^{* *}\left(\rho_{d}, b\right)}{\left[1+\beta_{d} G_{d-1}^{* *}\left(\rho_{d}, \rho_{d}\right)\right] \widehat{G}_{d-1}\left(\rho_{d}, \rho_{d}\right)} \\
=\frac{\widehat{G}_{d-1}\left(a, \rho_{d}\right) \widehat{G}_{d-1}\left(\rho_{d}, b\right)}{2\left[\widehat{G}_{d-1}\left(\rho_{d}, \rho_{d}\right)\right]^{2}\left[\beta_{d}+\frac{1}{\widehat{G}_{d-1}\left(\rho_{d}, \rho_{d}\right)}\right]}
\end{gathered}
$$

where $\widehat{G}_{d}(a, b)=G_{d}^{* *}(a, b)-G_{d}^{* *}(a,-b)$.

We have $\widehat{G}_{d}(a, b)=\widehat{G}(b, a), \widehat{G}_{d}(a, b)=\widehat{G}_{d-1}(a, b)-\beta_{d} \frac{\widehat{G}_{d-1}\left(a, \rho_{d}\right) \widehat{G}_{d-1}\left(\rho_{d}, b\right)}{1+\beta_{d} \widehat{G}_{d-1}\left(\rho_{d}, \rho_{d}\right)}$ and $\widehat{G}$ satisfies the 4 relations in the appendix of [12]. From now on, we use $\widehat{G}$ instead of $G^{* *}$, the proof follows Section 5.4.1 of [12] closely.

Proof of Lemma 3, Part (B): We must now analyze

$$
\bar{G}_{d}^{* *}(a, b)-\bar{G}_{d-1}^{* *}(a, b)=G_{d-1}^{* *}(a, b)-\bar{G}_{d-1}^{* *}(a, b)-\frac{\widehat{G}_{d-1}\left(a, \rho_{d}\right) \widehat{G}_{d-1}\left(b, \rho_{d}\right)}{2 \widehat{G}_{d-1}\left(\rho_{d}, \rho_{d}\right)} .
$$

This first part clearly leads to $\Theta(d-1)$.

The second part gives

$$
-\frac{\alpha^{d-1}}{2 F_{1}^{2}\left[C_{1}(d)+C_{2}(d) D_{2}(d) / F_{1}(d)\right]}
$$

\subsubsection{Using the random mapping approach}

Now we will use the results of Section 2.2 in order to deduce Theorem 4.1. The proof runs in the following way: First we apply Lemma 2.1 of [6] in order to get an asymp- 
totic expansion of $g\left(z, u_{1}, \ldots, u_{d}\right)$. Then we will apply Cauchy's formula and singularity analysis in the sense of Flajolet and Odlyzko [9]. We will omit details like error estimates, since this works in a very similar way as in [12]. There is also another way to get a more rigorous proof via the random mapping approach: When we consider random mapping built of planted plane trees instead of Cayley trees. Since this can be viewed as a special case of constrained random mappings (see $[3,4,11]$ ), it is easy to see that Theorem 2.1 still holds (with a different scaling parameter of course: $\sqrt{2}$ instead of 2). Thus the explicit formulas [12, Equations (31) and (32)] can be used instead of the asymptotic ones below and the error estimates are much easier. But on the other hand, dealing with those explicit expressions is much more involved and does not provide any deeper insight.

We have the following lemma.

Lemma 4: Assume that $\left|z-\frac{1}{e}\right| \rightarrow 0$ in such a way that $|a(z)| \leq 1+\circlearrowleft\left(\frac{1}{\sqrt{n}}\right)$, as $n \rightarrow \infty$, and $|u-a(z)|=\circlearrowleft\left(\frac{1}{\sqrt{n}}\right)$. Then we have the following asymptotic relation
uniformly for $r=\circlearrowleft(\sqrt{n}):$

$$
b_{r}(z, u)=\frac{A_{r}-u B_{r}}{C_{r}-u D_{r}}(1+\sigma((1-a(z))))
$$

where

$$
\begin{gathered}
A_{r}=\left(1+\frac{a(z)}{2} \frac{1-a(z)^{r}}{1-a(z)}-a(z)^{r}\right) a(z) \\
B_{r}=\frac{a(z)}{2} \frac{1-a(z)^{r}}{1-a(z)}-a(z)^{r} \\
C_{r}=1+\frac{a(z)}{2} \frac{1-a(z)^{r}}{1-a(z)} \\
D_{r}=\frac{1}{2} \frac{1-a(z)^{r}}{1-a(z)} .
\end{gathered}
$$

Proof: Set $\varphi(t)=e^{t}, \sigma=1$, and $\tau=1$ in Lemma 2.1 of [6]. Then the above form can be obtained by elementary algebra.

Using this expansion, we obtain after a straight forward calculation, the following formula: $\left[u_{1}^{k_{1}} \ldots u_{d}^{k}\right] g\left(z, u_{1}, \ldots, u_{d}\right)=\left[z^{n}\right] F(z)$ with

where

$$
\begin{gathered}
F(z)=\left(\frac{C_{r_{1}}}{C_{r_{1}}-A_{r_{1}}}-\frac{D_{r_{1}}}{D_{r_{1}}-B_{r_{1}}}\right)\left(\frac{D_{r_{1}}-B_{r_{1}}}{C_{r_{1}}-A_{r_{1}}}\right)^{k_{1}}\left(\frac{A_{h_{12}}}{C_{h_{12}}}\right)^{k_{1}}\left(\frac{D_{h_{d-1, d}}}{C_{h_{d-1, d}}}\right)^{k} S_{d} \\
\times \prod_{l=2}^{d-1}\left[\left(\frac{D_{h_{l-1, l}}}{C_{h_{l-1, l}}}\right)^{k_{l}}\left(\frac{A_{h_{l, l+1}}}{C_{h_{l, l+1}}}\right)^{k_{l}} S_{l}\right],
\end{gathered}
$$

$$
S_{l}=\sum_{i=0}^{\min \left(k_{l}-1, k_{l}\right)-1}\left(\begin{array}{c}
k_{l-1} \\
i+1
\end{array}\right)\left(\begin{array}{c}
k_{l}-1 \\
i
\end{array}\right)\left(1-\frac{B_{h_{l-1, l}} C_{h_{l-1, l}}}{A_{h_{l-1, l}} D_{h_{l-1, l}}}\right)^{i+1}
$$


Now, in order to calculate this coefficient, we use Cauchy's integral formula choosing a truncated line normal to the real axis and complemented by a circular arc as the integration path. To be precise, we integrate along $\Gamma=\gamma^{\prime} \cup \Gamma^{\prime}$ given by

$$
\begin{gathered}
\gamma^{\prime}=\left\{z: z=\frac{1}{e}\left(1-\frac{1+i t}{n}\right) \text { and }|t| \leq \sqrt{2 n+1}\right\} \\
\Gamma^{\prime}=\left\{z:|z|=\frac{1}{3} \text { and } \arctan \frac{\sqrt{2 n+1}}{n-1} \leq|\arg z| \leq \pi\right\} .
\end{gathered}
$$

On $\gamma^{\prime}$, we substitute $z=\frac{1}{3}\left(1-\frac{\alpha}{n}\right)$. Now using the well-known expansion for the tree function (see e.g., [19]) on $\gamma^{\prime}$

$$
a(z) \sim 1-\sqrt{\frac{2 \alpha}{n}}
$$

we obtain the asymptotic relations

$$
\begin{aligned}
& \frac{C_{r_{1}}}{C_{r_{1}}-A_{r_{1}}}-\frac{D_{r_{1}}}{D_{r_{1}}-B_{r_{1}}} \sim \frac{1}{\cosh ^{2}\left(\rho_{1} \sqrt{\alpha / 2}\right)} \\
& \left(\frac{D_{r_{1}}-B_{r_{1}}}{C_{r_{1}}-A_{r_{1}}}\right)^{k_{1}} \sim \exp \left(-y_{1} \sqrt{2 \alpha} \frac{\sinh \left(\rho_{1} \sqrt{\alpha / 2}\right)}{\cosh \left(\rho_{1} \sqrt{\alpha / 2}\right)}\right) \\
& \left(\frac{A_{h_{l, l+1}}}{C_{h_{l, l+1}}}\right)_{l}^{k} \sim \exp \left(-\frac{y_{l} \sqrt{2 \alpha} \cosh \left(\left(\rho_{l+1}-\rho_{l}\right) \sqrt{\alpha / 2}\right)}{\sinh \left(\left(\rho_{l+1}-\rho_{l}\right) \sqrt{\alpha / 2}\right)}\right) \\
& \left(\frac{D_{h_{l, l+1}}}{C_{h_{l, l+1}}}\right)_{l+1}^{k} \sim \exp \left(-\frac{y_{l} \sqrt{2 \alpha} \cosh \left(\left(\rho_{l+1}-\rho_{l}\right) \sqrt{\alpha / 2}\right)}{\sinh \left(\left(\rho_{l+1}-\rho_{l}\right) \sqrt{\alpha / 2}\right)}\right)
\end{aligned}
$$

The sum of $S_{l}$ can be approximated with the help of Stirling's formula and by extending the range of summation to infinity. For details see [12]. In this way, we get

$$
\begin{aligned}
& S_{l} \sim \frac{2 \alpha}{\sinh ^{2}\left(\left(\rho_{l}+1-\rho_{l}\right) \sqrt{\alpha / 2}\right)} \\
& \cdot \sqrt{\frac{y_{l} \sinh ^{2}\left(\left(\rho_{l+1}-\rho_{l}\right) \sqrt{\alpha / 2}\right)}{2 y_{l+1} \alpha}} I_{1}\left(2 \sqrt{\frac{2 \alpha y_{l} y_{l+1}}{\sinh ^{2}\left(\left(\rho_{l+1}-\rho_{l}\right) \sqrt{\alpha / 2}\right)}}\right)
\end{aligned}
$$

and inserting (17)-(21) into (16) and applying (8) yields (4.1) as desired.

Proof of Part $(\boldsymbol{B})$ : This part is immediate. One has just to compute the coefficients

$$
\begin{gathered}
{\left[z^{n} u_{1}^{0} \ldots u_{d}^{0}\right] g\left(z, u_{1}, \ldots, u_{d}\right)} \\
{\left[z^{n} u_{1}^{k} u_{2}^{0} \ldots u_{d}^{0}\right] g\left(z, u_{1}, \ldots, u_{d}\right)} \\
\vdots \\
{\left[z^{n} u_{1}^{k}{ }^{k} \ldots u_{d-1}^{k}{ }^{1} u_{d}^{0}\right] g\left(z, u_{1}, \ldots, u_{d}\right)}
\end{gathered}
$$


which is an easy exercise.

\section{References}

[1] Aldous, D., The continuum random tree II: An overview, In: Stochastic Analysis (ed. by T. Barlow and N.H. Bingham), Cambridge University Press (1991).

[2] Aldous, D. and Pitman, J., Brownian bridge asymptotics for random mappings, Random Struct. Alg. 5 (1994), 487-512.

[3] Arney, J. and Bender, E.A., Random mappings with constraints on coalescence and number of origins, Pacific J. Math. 103 (1982), 269-294.

[4] Baron, G., Drmota, M. and Mutafchiev, L., Predecessors in random mappings, Combin. Probab. Comput. 5 (1996), 317-335.

[5] Cohen, J.W. and Hooghiemstra, G., Brownian excursion, the M/M/1 queue and their occupation times, Math. Operat. Res. 6 (1981), 608-629.

[6] Drmota, M. and Gittenberger, B., On the profile of random trees, Rand. Str. Alg. 10 (1997), 421-451.

[7] Drmota, M. and Gittenberger, B., Strata of random mappings - a combinatorial approach, Stoch. Proc. Appl. (to appear).

[8] Drmota, M. and Soria, M., Marking in combinatorial constructions: generating functions and limiting distributions, Theoret. Comp. Sci. 144 (1995), 67-99.

[9] Flajolet, P. and Odlyzko, A.M., Singularity analysis of generating functions, SIAM J. Discr. Math. 3 (1990), 216-240.

[10] Flajolet, P. and Vitter, J.S., Average-case analysis of algorithms and data structures, In: Handbook of Theoretical Computer Sciences (ed. by J. van Leeuwen) A: Algorithms and Complexity, North Holland (1990), Chapt. 1, 431-524.

[11] Gittenberger, B., On the number of predecessors in constrained random mappings, Stat. Probab. Letters 36 (1997), 29-34.

[12] Gittenberger, B. and Louchard, G., The Brownian excursion multi-dimensional local time density, J. Appl. Probab. 36 (1999), 350-373.

[13] Hooghiemstra, G., On the explicit form of the density of Brownian excursion local time, Proc. AMS 84 (1982), 127-130.

[14] Itô, K. and McKean, Jr., H.P., Diffusion Processes and Their Sample Paths, Springer-Verlag, Berlin 1974.

[15] Knuth, D.E., The Art of Computer Programming, Addison-Wesley 1973.

[16] Kolchin, V.F., Random Mappings, Optimization Software, New York 1986.

[17] Louchard, G., Brownian motion and algorithm complexity, BIT 26 (1986), 17 34.

[18] Louchard, G., Kac's formula, Levy's local time and Brownian excursion, J. Appl. Prob. 21 (1984), 479-499.

[19] Meir, A. and Moon, J.W., On the altitude of nodes in random trees, Can. J. Math. 30 (1978), 997-1015.

[20] Mutafchiev, L., The limit distribution of the number of nodes in low strata of a random mapping, Stat. Prob. Letters 7 (1989), 247-251.

[21] Proskurin, G.V., On the distribution of the number of vertices in strata of a random mapping, Theory Probab. Appl. 18 (1973), 803-808.

[22] Takács, L., On the local time of the Brownian motion, Ann. Appl. Probab. 5 (1995), 741-756. 


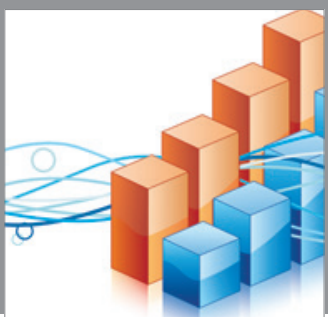

Advances in

Operations Research

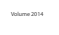

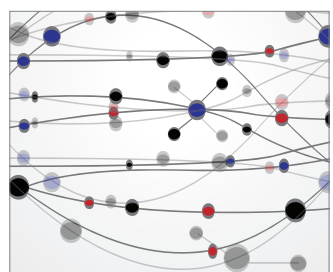

\section{The Scientific} World Journal
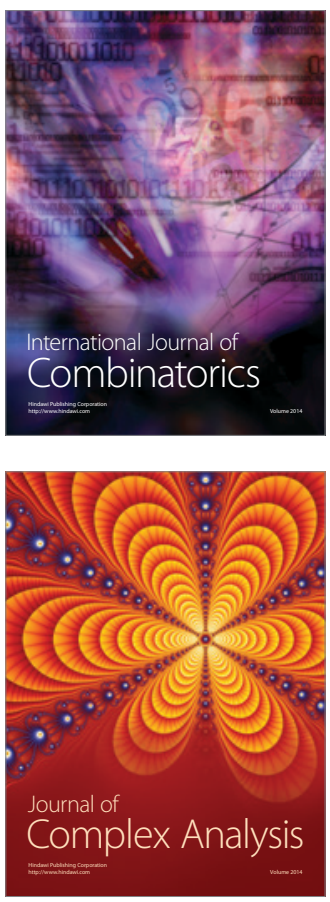

International Journal of

Mathematics and

Mathematical

Sciences
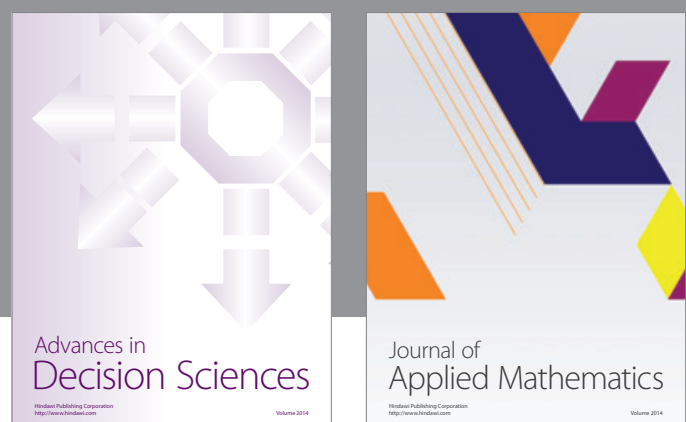

Journal of

Applied Mathematics
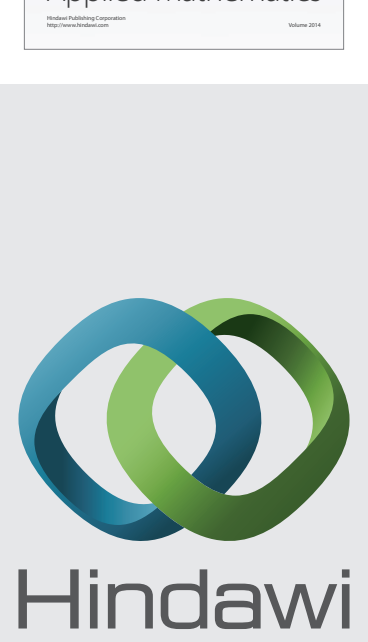

Submit your manuscripts at http://www.hindawi.com
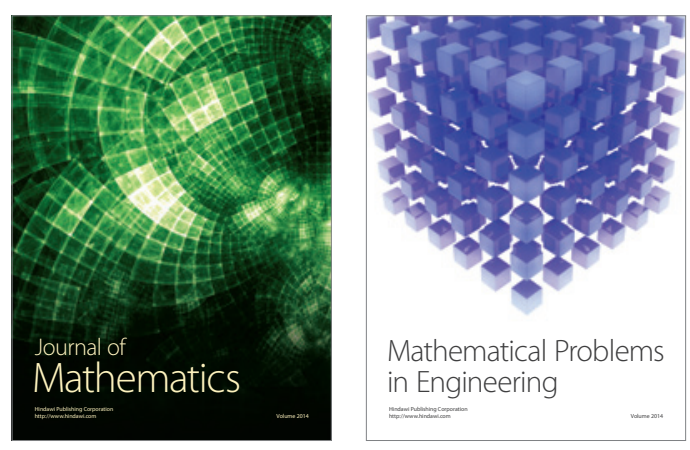

Mathematical Problems in Engineering
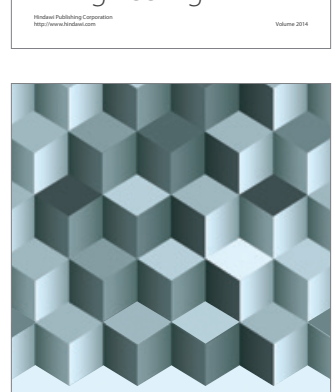

Journal of

Function Spaces
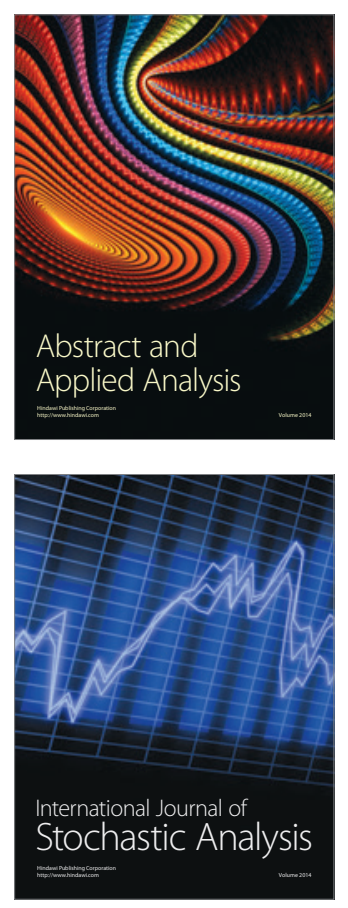

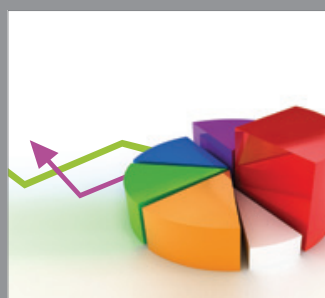

ournal of

Probability and Statistics

Promensencen
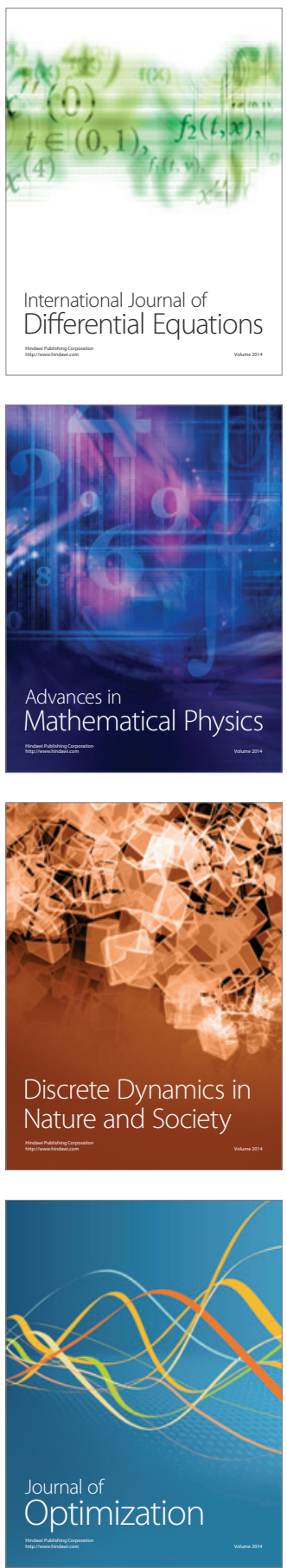